\title{
Testosterone therapy and cardiovascular events
}

\author{
C. Mary Schooling and Benjamin J. Cowling
}

Given the increasing use of testosterone in the USA and elsewhere, ${ }^{1-3}$ Spitzer et al. (Risks and benefits of testosterone therapy in older men. Nat. Rev. Endocrinol. 9, doi: $10.1038 /$ nrendo.2013.73 $)^{4}$ provide a timely assessment of its potential benefits and risks. The authors are cautious about testosterone therapy as a potential treatment for low testosterone levels in older men (defined as men aged $>65$ years) because, apart from lingering concerns about prostate cancer, testosterone therapy raises hematocrit and lowers HDLcholesterol levels, ${ }^{4}$ although this therapy can also improve exercise capacity among men with heart failure. ${ }^{5}$

Spitzer et al. suggest large prospective randomized trials to establish the effects of testosterone therapy on cardiovascular health. Such trials may be the best way to determine the effects of testosterone on the leading cause of mortality and morbidity, where even a very small change in risk would have a large effect on population health. On the other hand, results of such prospective trials, similar to the Women's Health Initiative trial, ${ }^{6}$ for example, can take a decade to obtain; however, use of testosterone is increasing rapidly now. ${ }^{1-3}$ Consideration of all the evidence to date should guide policy in the interim.

No large trial has ever been implemented to assess the effect of testosterone on cardiovascular health. Meta-analysis of small trials might be more reliable than a large trial. ${ }^{7}$ Spitzer et al. report a pooled odds ratio of about 1.8 (95\% CI 0.8-4.2) for the effect of testosterone therapy on cardiovascular events in their Figure $3,{ }^{4}$ the data for which is taken from two meta-analyses published in $2005^{8}$ and $2007^{9}$ of trials reported before 2005. ${ }^{8,9}$ How the authors obtained this estimate is not described, although one of the meta-analyses reported an odds ratio of 1.82 (95\% CI $0.78-4.23$ ) based on 21 events among 308 men from six trials, whilst also reporting that a total of 485 men participated in these six trials (average age 62 years). ${ }^{9}$ Assuming this estimate is a reliable summary of a representative sample of all possible trials reported before 2005 and that the effects do not vary with age, ${ }^{10}$ a synthesis of all trials to date would now be expected to give a similar estimate with greater precision and potentially with CI values $>1$.

We recently published an updated metaanalysis, which included 27 trials investigating the effects of testosterone therapy on 180 cardiovascular-related events among 2,994 mainly older men (average age 62 years). ${ }^{11}$ The pooled odds ratio for testosterone therapy on cardiovascular-related events was 1.54 (95\% CI 1.09-2.18), with a higher event rate and odds ratio for trials not funded by the pharmaceutical industry. ${ }^{11}$ Of course, existing on-going trials may show testosterone to be protective against cardiovascular disease and thereby reduce the estimate of harm; however, this hypothesis would be against the run of evidence to date.

On the basis of the current evidence, any large prospective randomized trial to definitively establish the effects of testosterone on cardiovascular health should perhaps be of an agent that reduces testosterone, rather than administration of testosterone. Notably, one such agent, statins, ${ }^{12}$ is already in widespread use to treat cardiovascular disease, but other suitable agents might also exist. Such trials could also assess whether any effects on cardiovascular disease are, at least in part, mediated by their effect on testosterone.

CUNY School of Public Health at Hunter College, 2180 Third Avenue, New York, NY 10035, USA (C. M. Schooling). School of Public Health, Li Ka Shing Faculty of Medicine, The University of Hong Kong, 21 Sassoon Road, Pokfulam, Hong Kong, China (B. J. Cowling). Correspondence to: C. M. Schooling mschooli@hunter.cuny.edu
Competing interests

B. J. Cowling declares an association with the following companies: Crucell, Medlmmune. See the article online for details of the relationships. C. M. Schooling declares no competing interests.

1. [No authors listed] Sexual dysfunction as the last bastion of urological drug commercialisation within the pharmaceutical industry. BJU Int. 107, 1845-1846 (2011).

2. Handelsmann, D. J. Pharmacoepidemiology of testosterone prescribing in Australia, 1992-2010. Med. J. Aust. 196, 642-645 (2012).

3. Gan, E. H., Pattman, S., Pearce, S. $\&$ Quinton, R. Many men are receiving unnecessary testosterone prescriptions. BMJ 345, e5469 (2012).

4. Spitzer, M., Huang, G., Basaria, S., Travison, T. G. \& Bhasin, S. Risks and benefits of testosterone therapy in older men. Nat. Rev. Endocrinol. http://dx.doi.org/10.1038/ nrendo.2013.73.

5. Toma, M. et al. Testosterone supplementation in heart failure: a meta-analysis. Circ. Heart Fail. 5, 315-321 (2012).

6. Rossouw, J. E. et al. Risks and benefits of estrogen plus progestin in healthy postmenopausal women: principal results from the Women's Health Initative randomized controlled trial. JAMA 288, 321-333 (2002).

7. Inthout, J., Ioannidis, J. P. \& Borm, G. F. Obtaining evidence by a single well-powered trial or several modestly powered trials. Stat. Methods Med. Res. http://dx.doi.org/ 10.1177/0962280212461098.

8. Calof, O. M. et al. Adverse events associated with testosterone replacement in middle-aged and older men: a meta-analysis of randomized, placebo-controlled trials. J. Gerontol. A. Biol. Sci. Med. Sci. 60, 1451-1457 (2005).

9. Haddad, R. M. et al. Testosterone and cardiovascular risk in men: a systematic review and meta-analysis of randomized placebocontrolled trials. Mayo Clin. Proc. 82, 29-39 (2007).

10. Petitti, D. Commentary: hormone replacement therapy and coronary heart disease: four lessons. Int. J. Epidemiol. 33, 461-463 (2004).

11. Xu, L., Freeman, G., Cowling, B. J. \& Schooling, C. M. Testosterone therapy and cardiovascular events among men: a systematic review and meta-analysis of placebo-controlled randomized trials. BMC Med. 11, 108 (2013).

12. Schooling, C. M., Au Yeung, S. L., Freeman, G. \& Cowling, B. J. The effect of statins on testosterone in men and women, a systematic review and meta-analysis of randomized controlled trials. BMC Med. 11, 57 (2013). 